\title{
Prediction of Fake Tweets Using Machine Learning Algorithms
}

\author{
M. Sreedevi ${ }^{\text {a, } 1}$, G. Vijay Kumar ${ }^{\text {a }}$, K. Kiran Kumar ${ }^{\text {a }}$, B. Aruna ${ }^{\text {a }}$, Arvind Yadav ${ }^{\text {a }}$ \\ a Department of Computer Science \& Engineering, Koneru Lakshmaiah Education \\ Foundation, Greenfields, Vaddeswaram, Guntur-522502, India
}

\begin{abstract}
Social networking sites will attract millions of users around the globe. Internet media is becoming popular for news consumption because of its ease, simple access and fast spreading of data takes to consume news from social media. Fake news on social media is making an appearance that is attracting a huge attention. This kind of situation could bring a great conflict in real time. The false news impacts extremely negative on society, particularly in social, commercial, political world, also on individuals. Hence detection of fake news on social media became one of the emerging research topic and technically challenging task due to availability of tools on social media. In this paper various machine learning techniques are used to predict fake news on twitter data. The results shown by using these techniques are more accurate with better performance.
\end{abstract}

Keyword. Social media, networking, Fake tweets, Data mining, Prediction

\section{Introduction}

In recent years, the popularity of social media has increased significantly because many people give priority to read the news through social media [1]. There are millions of news content published on the internet than traditional news paper, so social media became the main platform in terms of spreading news. However, Twitter App [2] is not only based on individual opinions, and also for an official information of a particular occasion could be delivered, such as organization could use Twitter-API like an unique and personal $[3,4]$ user. There are variety of news can spread over social network such as satire, Hoax and fabrications. Hoax is one type of false news can spread using various sources like stories, people will easily trust this type of news. Satire is another type of false news where true is presented as a joke. So there is a chance to consider the real news as silly joke. Fabrications are international lies, these are depends on click bytes [5].

Twitter can likewise might be utilized in a different term, and it is worth might be boundless later. Knight Foundation began an examination to explore how counterfeit news spread on the stage previously, during and after the 2016 U.S. official political decision [1]. The assessment broke down more than a huge number of tweets from many Twitter accounts that connected to in excess of 600 phony and intrigue news destinations.

\footnotetext{
${ }^{1}$ M. Sreedevi, Department of Computer Science \& Engineering, Koneru Lakshmaiah Education Foundation Email: 170030759@kluniversity.in.
} 
It recognized bunches of Twitter accounts that connected back to these destinations more than once, regularly in manners that appeared to be composed or even mechanized. Fake news is duplicate or a deceptive data introduced as news. It regularly has a point of harming the standing of an individual. First, fake news [6] is 8 intentionally written to mislead consumers, it does not make satisfactory to spot fake news from news itself.

You can report straightforwardly from an individual Tweet, List, or profile for specific infringement, including spam, oppressive or hurtful substance, wrong advertisements, self- mischief, and pantomime. For data about announcing different sorts of infringement but even to report the spam issues or fake tweets [7] we need to identify the fake tweets so to predict such fake information we are about to use 5 algorithms [6] and it would be helpful to report all kinds of harmful rumors.

\section{Literature Survey}

In [8] Author suggested design of four modules are Feature Clustering using Modified K- means, user interface, log pre-processing, Naïve Bayes Classification testing and training using k-nearest neighbors' algorithm for best Précised classification of viewpoint. The design could find unrelated data and more accuracy using $\mathrm{K}$ means Modified with Naïve Bayes algorithm. Present research is searching on Sentimental analysis [9] regarding complete decision of highly organized web sources such as communication groups like gatherings, feedbacks are in digital form. Many ways that got improved for main problems of opinion mining's and got solved. We received a picture of which are involved in making a software system for sentimental analysis regarding the analysis. In the calculation investigates the connection got and returns a string message that incorporates the Decision, the User score, and the Tweet Score. The twitter connect is incorporated of twitter address, client screen name and status id (tweet id). Twitter has an easy-to-understand way of taken this tweet connect. Then, calculation fills in as it follows: It gets the client subtleties from our information base, If they don't have the client in the twitter stockpiling, it gets its qualities from twitter and introduce its score to nothing, It gets the tweet's subtleties from Twitter, Creates a tweet score code dependent on the got information from various API's, Creates a client score code as a number juggling mean of its earlier score and genuine tweet score.

In [10] This paper presents a down to earth examination to pick a high perform characterization technique and the unfeasible purposes behind the high performed arrangement. They introduced their framework which work to recognize counterfeit clients and phony news in the Twitter interpersonal organization by acquiring validity score. The calculation at that point utilizes a Named Entity Recognition segment, what parts the content into its after parts: it draws out the marks, the subjects, the social labels, the general tweet assessment, and the hash-label opinion. Framework engineering For the NER parsing, they utilized a public API (Application Programming Interface) called Open-Calais, while for the opinion calculation, we utilized Sentiment140. In [11] polarity of text data identification in NLP became complex due to collection of feedback about products from various resources. To avoid this complexity they performed sentiment analysis by analyzing the behavior and attitude of people by using genetic algorithm using CNN for opinion classification. They studied 
existing sentiment analysis methods on twitter data and they provide comparisons theoretically of state-of-art approaches. They also analyzed document level sentiment analysis on twitter data using various methods. In [12] they performed classification approach using TSVM/SVM and C-SVM data to detect spam tweets or duplicate tweets on twitter data. Text performance on integrated cluster classification performed better results than SVM classification. Also, regular and popular pattern mining [13-15] are very much useful in prediction of fake tweets.

\section{METHODOLOGY}

To predict the given news is real or fake news, we used classification algorithms like Logistic regression, Naive bayes, Stochastic Gradient Descent (SGD), Support Vector Machine (SVM) and Random Forest. After evaluating the performance of these algorithms, we conclude which among these algorithms is the best.

Data Preprocessing: Data preprocessing is one of a data mining procedure which converts the raw data into understandable, useful, and efficient formats. True information is inadequate, conflicting and it contains many errors which affects the results. So, all these issues can be resolved by performing data preprocessing.

Data Cleaning: Data cleaning is the way toward eliminating off base, copy, or inadequate information inside a dataset. When we combine multiple information sources there are numerous chances for data to be duplicated.

Data Integration: Data Integration is information pre-processing procedure that joins data from multiple sources into a reasonable data and provides unified view of the data. Data Transformation: Data transformation is a data preprocessing technique that converts source framework into the necessary configuration of a destination system. It is the process of changing the format, structure or values of data.

Data Reduction: Data reduction is a data preprocessing strategy that decreases the measure of limit required to store the data. It can decrease the costs and increase storage capacity.

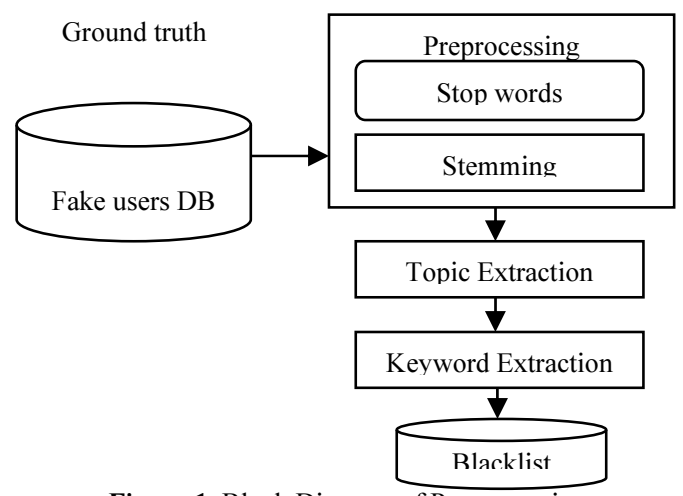

Figure 1. Block Diagram of Preprocessing

Logistic Regression: It helps to find the probability of success and failure of an event. It is a statistical model that in its basic form it models a binary dependent variable using logistic function. It is performed at the point when the needy variable is twofold. It is named as calculated relapse since it is very similar to direct relapse. The term 
'strategic' is taken from the capacity logit which is utilized in the technique for grouping. In regression analysis, logistic regression is making an estimation of the parameters of a logistic model.
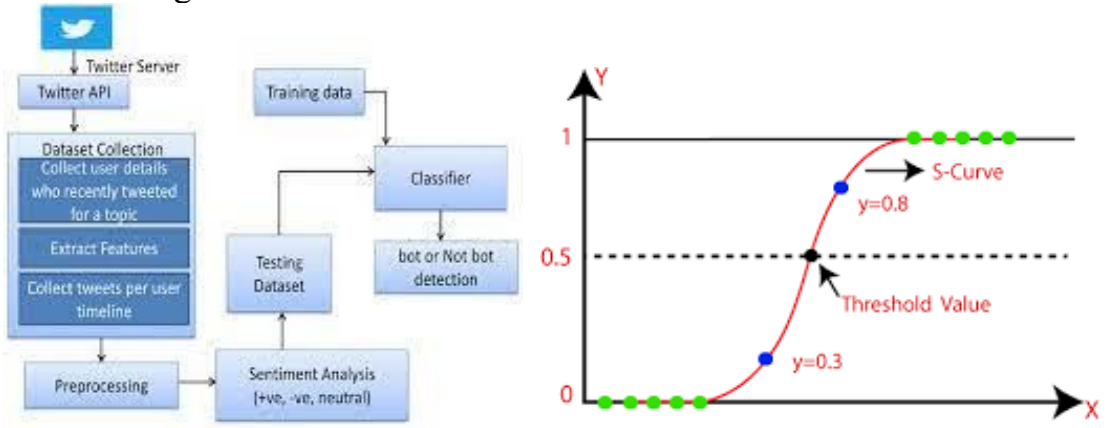

Figure 2. Process of Training data in Sentimental Analysis

Naive Bayes: Naive Bayes is used to predict the probability of different classes on various attributes. This can be utilized in text classification and it is used for problems having multiple classes. It is not sensitive to irrelevant features. It takes care of continuous and discrete information and it is mostly adaptable with number of highlights and data points. It makes real time predictions fast.

Stochastic Gradient Descent (SGD): The term 'stochastic' means a process that is linked with random probability. Stochastic gradient descent would randomly pick samples and uses the samples to calculate the derivatives. A couple of tests are randomly chosen instead of whole dataset for each iteration. It is especially useful when there are redundancies in the data.

Support Vector Machine (SVM): It is a supervised learning technique that can be utilized for relapse and order issues. It represents the datasets as points. The main objective of svm is to construct a hyperplane that divides the datasets into distinct categories. The hyperplane is a choice limit that helps in grouping information focuses, and the hyperplane ought to be at the limit edge from particular classes. This algorithm helps in removing the function that is too closely fit to a limited set of data points of the samples and provides better accuracy.

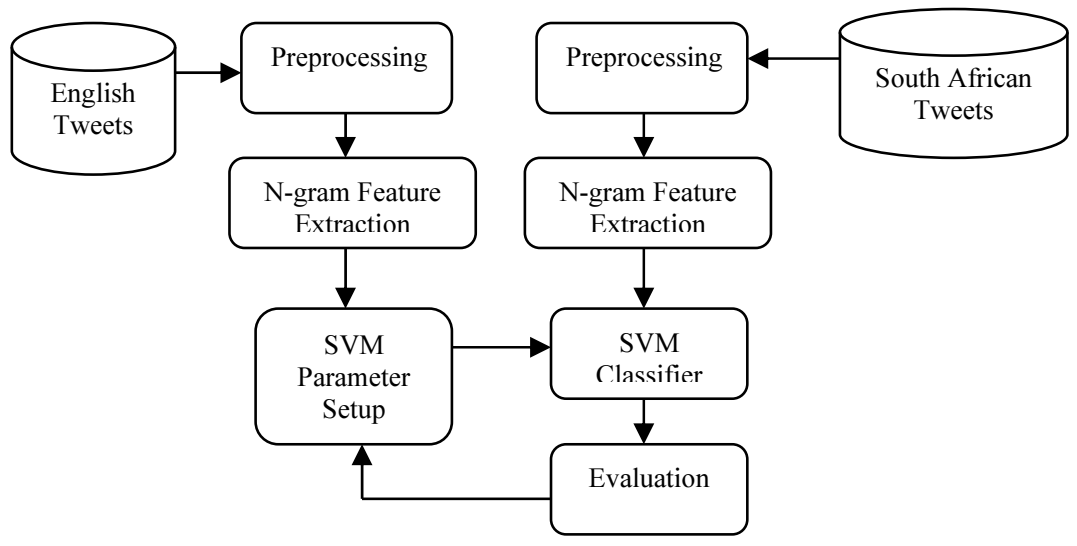

Figure 3. Comparison of various tweets in preprocessing

Random Forest: This is an outfit learning strategy for grouping and relapse that utilizes 
different models of a few choice trees to get better forecast execution. It constructs various choice trees and join them to get exact and stable expectations. It runs productively on huge data sets. It can deal with many information factors without taking out factor and gauge factors which are significant in the arrangement.

\section{Results}

Sample datasets are collected from Kaggle and we used python as working environment. After preprocessing the information is divided into train and test datasets. By using five different machine learning algorithms the following accuracy values are found to identify the news as fake or not is given in table 1 . The least accuracy is given by Naïve Bayes and highest accuracy is given by SVM about $99.48 \%$ and SGD is about $99.28 \%$. The values which got for prediction of fake news for 5 data mining algorithms are represented as line graph in figure 5.

Table 1. Algorithm Results

\begin{tabular}{|c|l|c|}
\hline S.No. & \multicolumn{1}{|c|}{ Model } & Accuracy \\
\hline 1 & Logistic Regression & $98.0 \%$ \\
\hline 2 & Naïve Bayes & $94.0 \%$ \\
\hline 3 & Stochastic Gradient Descent & $99.28 \%$ \\
\hline 4 & Support Vector Machine & $99.48 \%$ \\
\hline 5 & Random Forest & $98.0 \%$ \\
\hline
\end{tabular}

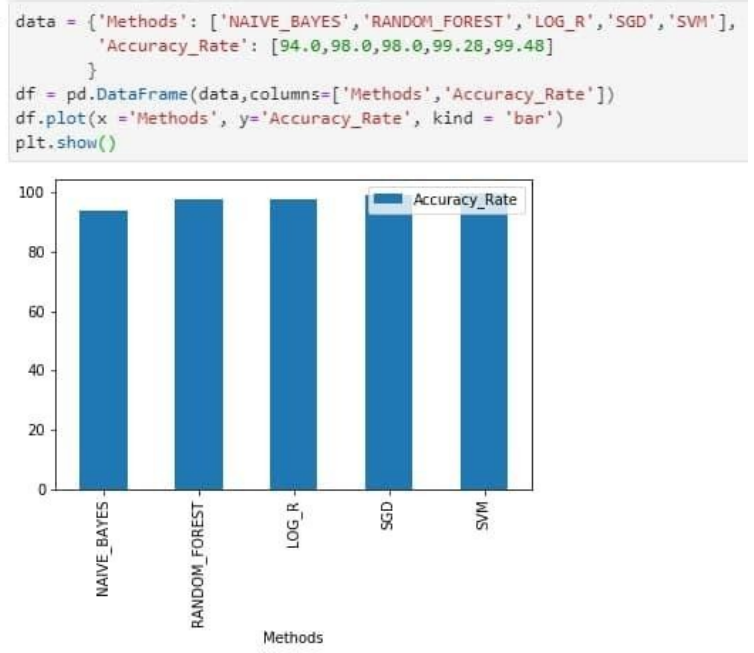

Figure 4. Accuracy Rate results on various algorithms

\section{Conclusion and Future Work}

In our project, we worked upon models to determine the fake news among the datasets using data mining techniques, and this paper had about five different classification models which are compared by the accuracies. SVM got the highest accuracy and it is about $99.48 \%$. In this paper we consider only twitter data for prediction of fake tweets. Our future scope is to predict fake 
news against political affairs, economic affairs and Health affairs on facebook and other social sites data.

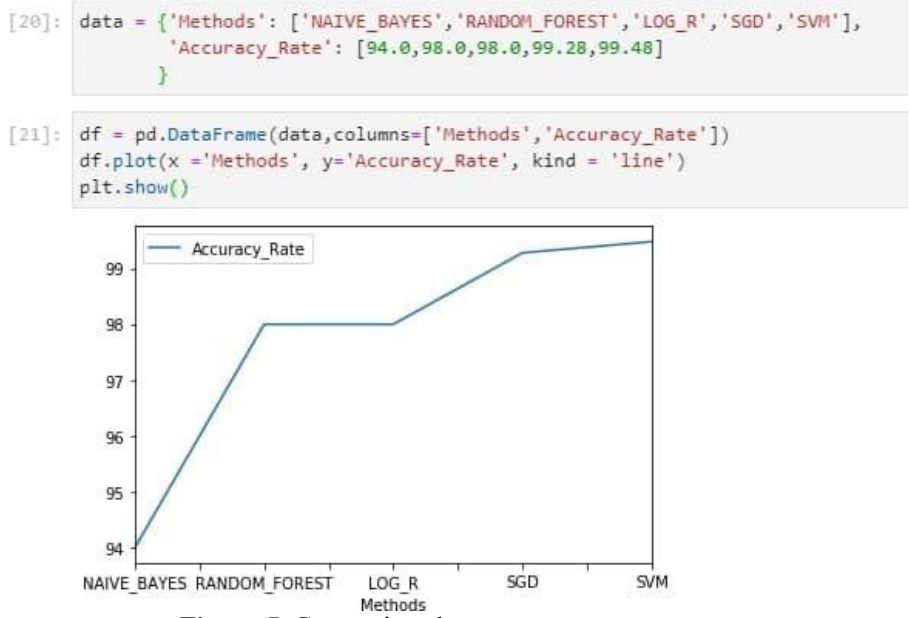

Figure 5. Comparison between accuracy rates

\section{References}

[1] Kelly Stahl, "Fake news detection in social media " B.S. Candidate, Department of Mathematics and Department of Computer Sciences, California State University Stanislaus, 1 University Circle, Turlock, CA (Confidentiality Agreement) 95382 Received 20 April, 2018; accepted 15 May 2018.

[2] Monika Verma, Sanjeev Sofat,"Techniques to Detect Spammers in Twitter- A Survey ", Int. J. Comput. Appl 85(10):27-32, January 2014.

[3] Costel-Sergiu Atodiresei*, Alexandru Tănăselea, Adrian Iftene , "Identifying FakeNews and Fake Users on Twitter " Available online 28 August 2018. Published by: ElsevierLtd.

[4] Potharaju, S. P., \& Sreedevi, M. (2017). A Novel Clustering Based Candidate Feature Selection Framework Using Correlation Coefficient for Improving Classification Performance. Journal of Engineering Science \& Technology Review, 10(6).

[5] Pallavi B. Petkar, S. S. Sonawane, "Fake News Detection: A Survey of Techniques", International Journal of Innovative Technology and Exploring Engineering (IJITEE) ISSN: 2278-3075, Volume-9 Issue-9, July 2020.

[6] Diana Elizabeth Roy, Prannay S Reddy, "A Study on Fakenews Detection Using Naïve Bayes, SVM (Support Vector Machine), Neural Networks and LSTM (Long Short-Term Memory).", Journal of Advanced Research in Control \& Dynamical Systems, 2019.

[7] Shlok Gilda, "Evaluating M L Algorithms for Fake News Detection" Student Conference on Research and Development (SCOReD), December 2017, pp.110-115.

[8] Potharaju, S. P., Sreedevi, M., \& Amiripalli, S. S. (2019). An Ensemble Feature Selection Framework of Sonar Targets Using Symmetrical Uncertainty and Multi-Layer Perceptron (SUMLP). In Cognitive Informatics and Soft Computing (pp. 247-256). Springer, Singapore.

[9] Abdullah Alsaeedi , Mohammadd Zubair Khan, "A study on sentiment Analysis Techniques of Twitter Data" Published in IJACSA, Volume 10 Issue 2, 2019.

[10] SreeDevi, M Rama Bai, M Chandrashekar Reddy."Newspaper Article Classification using Machine Learning Techniques"ijitee.March 2020

[11] Sravya, G., Sreedevi, M. "Genetic optimization in hybrid level sentiment analysis for opinion classification", International Journal of Advanced Trends in Computer Science and Engineering, 2020, 9(2), pp. 1440-1445.

[12] Laxmi Narasamma, V., Sreedevi, M. "A Comparative Approach for Classification and Combined Cluster based Classification Method for Tweets Data Analysis", Smart Innovation, Systems and Techologies 2020, pp. 329-339. 
[13] Vijay Kumar G, Sreedevi M., et al., "Incremental mining of popular patterns from transactional databases", IJET, vol 7 issue 2.7, pp. 636-641, 2018

[14] Vijay Kumar G, Sreedevi M et al., "Regular frequent crime pattern mining on crime datasets" IJET, vol 7 issue 2.7, pp. 972-975, 2018.

[15] Vijay Kumar G, Krishna Chaitanya T. "Mining Popular Patterns from Multidimensional Database", Indian Journal of Science and Technology, Vol 9 issue 17, May 2016. 